\title{
Coordinated Variation of Contact Angles During Mobilization of Double Liquid-Gas Interfaces in a Microcapillary
}

\author{
Da Lei ${ }^{1} \cdot$ Mian $\operatorname{Lin}^{2,3} \cdot$ Yun $\mathrm{Li}^{1} \cdot$ Wenbin Jiang ${ }^{2}$
}

Received: 6 May 2019 / Accepted: 18 January 2020 / Published online: 5 February 2020

(c) Springer Nature B.V. 2020

\begin{abstract}
Effectively mobilizing displacement and predicting mobilization pressure in a porous-type reservoir filled with bubbles or blobs require the knowledge of variation of contact angles and capillary pressure. A bubble/blob has two interfaces and thus has two contact angles. It has been found that double interfaces cause resistance to displacement, and the resisting pressure rises while one contact angle increasing and the other decreasing during mobilization. To quantitatively explain how the resistance to flow builds up according to the contact angle variations during mobilization, it is assumed that (1) contact points remain unmoved; (2) the volume of a bubble or blob maintains constant; (3) once the interface starts moving at low capillary number, the contact angle remains to be the advancing or receding angle; (4) the viscous effect on pressure drop can be ignored; and (5) the two angles of two interfaces are equal to an equilibrium angle at the initiation of mobilization. A theoretical model is developed based on these assumptions, and the quantitative relationship of the two angles is expressed by an implicit function. Combining Young-Laplace equation, the capillary pressure induced by double interfaces is obtained. The model's prediction is in good agreement with experiments in studies. The equilibrium angle has strong influence on the variation of the two angles. When the equilibrium angle is less than 90 degrees, a relatively greater change in the contact angle at the advancing interface leads to a smaller change in the other one. Otherwise, the opposite is true. The changes of the two angles are equal when the equilibrium angle is 90 degrees. Moreover, a linear trend proposed by a previous investigation is incorporated into the model, to predict the ending of mobilization stage and to predict the maximum mobilization pressure on a given solid surface.
\end{abstract}

Keywords Bubble/blob mobilization · Microscale capillary $\cdot$ Double interfaces $\cdot$ Contact angle hysteresis

Mian Lin

linmian@imech.ac.cn

Yun Li

yunli@mail.xjtu.edu.cn

1 School of Chemical Engineering and Technology, Xi'an Jiaotong University, Xi' an 710049, Shaanxi, China

2 Institute of Mechanics, Chinese Academy of Sciences, Beijing 100190, China

3 School of Engineering Science, University of Chinese Academy of Sciences, Beijing 100049, China 


\section{Introduction}

Mobilization of liquid-gas interfaces in a capillary is closely related to industrial applications like oil recovery from porous-type reservoirs with water-alternating-gas injection, solution gas drive and other enhanced oil recovery technologies (Bora et al. 2000; Sohrabi et al. 2004), also related to the intrusion of liquid into membranes or fibrous media (Moghadam et al. 2018; Jamali et al. 2018). Moreover, a dispersed phase like air bubbles, oil blobs (either trapped by air or other immiscible liquids) can be found distributed across the porous media during the two-phase displacement, indicating that advancing and receding of interfaces can occur simultaneously (Morrow et al. 1988; Bora et al. 2000; Pak et al. 2015). These entrapped bubbles or blobs can build up substantial resistance to the flow and significantly influence the relative permeabilities (Taber 1980; Morrow et al. 1988). Efficiently mobilizing the displacement is of principal economic interest to the petroleum industry (Hsu et al. 2012).

Most pore-scale displacement occurs under low capillary numbers $\mathrm{Ca}(\mathrm{Ca}=\mu \mathrm{V} / \gamma$, where $\mu$ is the viscosity of the liquid, $V$ the interface velocity and $\gamma$ the interfacial tension) ranging from $10^{-10}$ to $10^{-5}$ and Bond numbers $B o\left(B o=\rho g h^{2} / \gamma\right.$, where $\rho$ is the density of the liquid, $g$ the gravitational constant and $h$ the characteristic length of the flow) less than $10^{-4}$, indicating that the dominating role of capillary pressure and the capillary diameter is less than $30 \mu \mathrm{m}$ (Piri and Blunt 2005). The behavior of a single interface is the basis of double interfaces mobilization. Experiments found that in a capillary a single interface moves forward when the contact angle reaches an advancing angle, and moves backward when the contact angle reaches a receding angle (Morrow and Nguyen 1982; Lei et al. 2018). The same phenomenon is also observed in liquid bridge experiments (Chen et al. 2013). The contact angle remains constant when moving with $10^{-9}<C a<10^{-5}$, and the capillary pressure of an interface can either resist or assist its movement, depending on the contact angle (Lei et al. 2018).

When forming a bubble/blob, will the total capillary pressure of double interfaces also resist or assist the movement depending on the contact angles? There are also experiments regarding multiple interfaces within a capillary forming bubbles or blobs. It is found that air bubbles in microtubes can sustain a certain amount of pressure, which is known as the Jamin effect and was ascribed to the contact angle hysteresis (Smith and Crane 1930; Gardescu 1930; Uchiyama et al. 2015). The number of bubbles in a capillary has been found to positively affect the pressure needed to mobilize the displacement (Smith and Crane 1930; Uchiyama et al. 2015). But the length of a bubble does not influence the pressure needed to initiate the movement (Srinivasan et al. 2013, 2015). In experiments of mobilizing air bubbles, constant injection rate is often used and the measured data reveal that the pressure drop of bubbles keeps rising until bubbles start moving (Srinivasan et al. 2013, 2015; Uchiyama et al. 2015). Srinivasan et al. (2013) managed to measure the pressure and contact angles during bubble mobilization in a 1.5 -mm-diameter capillary. During mobilization, the contact angle at the advancing interface increases and the other at the receding interface decreases as the rising of pressure drop.

Despite the experimental measurement and observations, there is still a lack of quantitatively description of the variation of contact angles or capillary pressure of double interfaces during mobilization.

This work focuses on how the resistance to flow is building up during mobilization of a bubble or blob, according to the variation of contact angles. A model is developed to describe the coordinated variation of contact angles of double liquid-gas interfaces in 
a microcapillary. Combining Young-Laplace equation, the capillary pressure of double interfaces can be further obtained. The model presented in this work is in good agreement with experiments in the literature. Furthermore, in order to predict the maximum mobilization pressure on a given solid surface, an empirical advancing/receding angle relationship is incorporated into the presented model.

\section{Theory Basis}

\subsection{Mobilization of a Single Interface}

A bubble or blob within a capillary consists of two interfaces. Understanding the mobilization of a single interface is the bridge to understanding the mobilization of double interfaces. As defined in the movement of a single interface, when the liquid-gas interface advances, it moves toward liquid side; when the liquid-gas interface recedes, it moves toward gas side. Thus, a bubble or blob has an interface that tends to advance and the other tends to recede.

It is widely acknowledged that there are two limiting values of the metastable apparent contact angle when the contact point is on the verge of advancing or receding, which is commonly attributed to solid surface heterogeneities (Bonn et al. 2009; Ramiasa et al. 2014). The maximum metastable apparent angle (threshold angle) is the advancing angle $\theta_{\text {adv }}$, and the minimum is the receding angle $\theta_{\text {rec }}$; the difference between $\theta_{\text {adv }}$ and $\theta_{\text {rec }}$ is also referred to as contact angle hysteresis (CAH) (Marmur et al. 2017). Experiments found that hysteresis occurs the velocity of contact points are below the minimum measured velocity, such as $0.135 \mu \mathrm{m} / \mathrm{s}$ in latest experiments (Lei et al. 2018). Contact points can be thus regarded as unmoved. Figure 1 demonstrates the hysteresis process of a glycerol-air meniscus in a microcapillary. In Fig. 1, the liquid is pushed to advance and the contact angle keeps increasing, while the contact points show no obvious movement.

After the contact angle reaches beyond $\theta_{\text {adv }}$, or gets less than $\theta_{\text {rec }}$, the equilibrium state is ended and the contact points start moving. Previous investigations confirm that contact angle during the movement of contact points remains constant at low capillary numbers, which cover the range of $\mathrm{Ca}$ from $10^{-9}$ to $10^{-5}$, summarizing all experiments (Johnson et al. 1977; Morrow and Nguyen 1982; Kwok et al. 1998; Lei et al. 2018). The capillary number of $10^{-9}-10^{-5}$ is also the range where most pore-level displacement occurs (Piri and Blunt 2005), as aforementioned. Hence, the dynamic contact angle $\theta_{d}$ can be represented by an advancing angle $\theta_{\text {adv }}$ and $\theta_{\text {rec* }}$.

$$
\begin{cases}\theta_{d}=\theta_{\mathrm{adv}} & C a>0 \\ \theta_{d}=\theta_{\mathrm{rec}} & C a<0\end{cases}
$$

The sign of $\mathrm{Ca}$ represents the direction of interface movement, positive for advancing and negative for receding. Artificial smooth surfaces (the root-mean-square roughness $<1 \mathrm{~nm}$ measured by atomic force microscopy) created by coating techniques could reduce the hysteresis of certain liquids to several degrees (Schneemilch et al. 1998; Petrov et al. 2003; Ramos and Tanguy 2006). However, in pores of tight reservoirs or other natural porous media without artificial refinement (i.e., coating, polishing), a significant difference between $\theta_{\text {adv }}$ and $\theta_{\text {rec }}$ of any liquid is expected. 


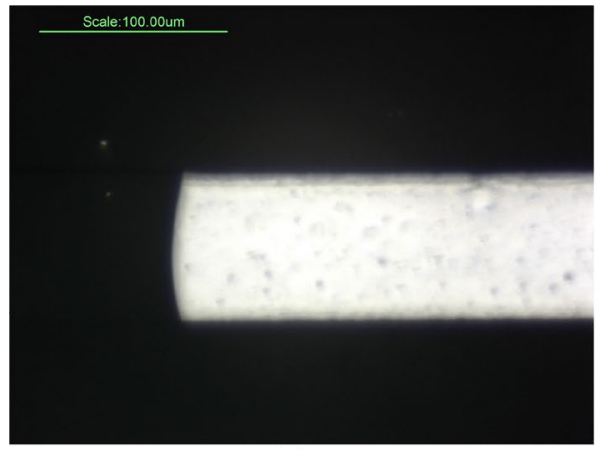

(a)

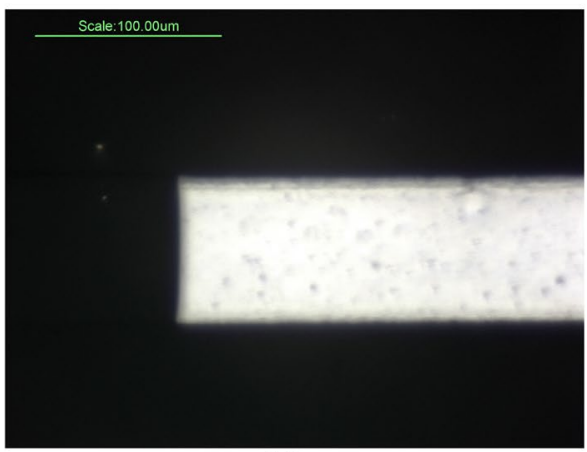

(c)

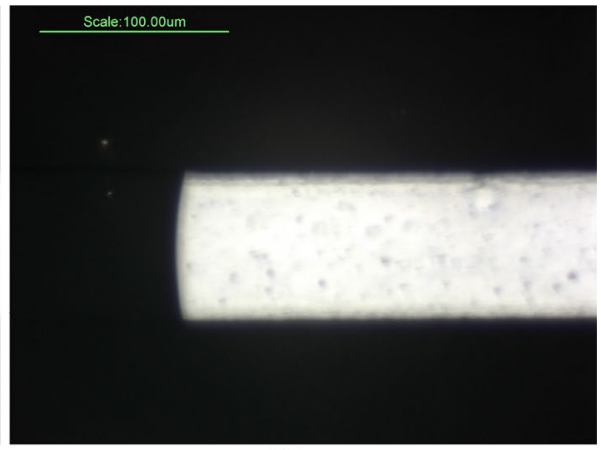

(b)

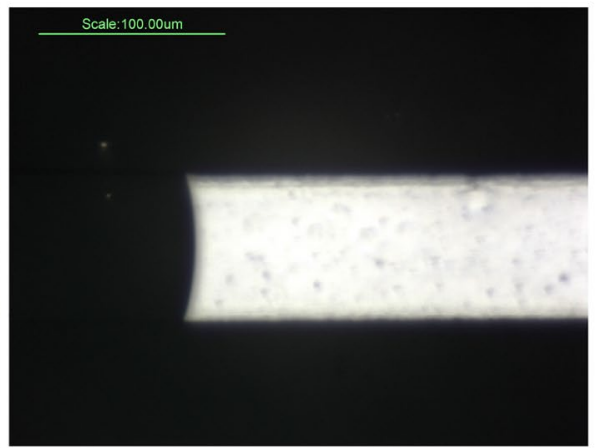

(d)

Fig. 1 Contact angle hysteresis of glycerol in an $80 \times 20$ (width $\times$ depth) $\mu \mathrm{m}$ rectangular capillary when the liquid is pushed to move to the right and contact points are stationary. The deformation of meniscus is in the sequence of (a-d). The original images can be found in Appendix of a previous investigation (Lei et al. 2018)

The contact angle hysteresis of a single interface and the velocity independence of contact angle at low capillary number lay the foundation of analyzing the mobilization of double interfaces.

\subsection{Mobilization of Double Interfaces}

\subsubsection{Model Assumptions}

Based on the behavior of a single liquid-gas interface in a microcapillary, several conclusions and assumptions can be utilized:

1. Contact points remain unmoved during hysteresis. The transition from advancing angle to receding angle occurs below the minimum measured velocity in different experiments, such as $2.5 \mathrm{~mm} / \mathrm{min}$ in the experiments of Johnson et al. (1977), $0.1 \mathrm{~mm} / \mathrm{min}$ in the experiments of Kwok et al. (1998) and $0.135 \mu \mathrm{m} / \mathrm{s}$ in the experiments of Lei et al. (2018). Thus, the contact point can be taken as unmoved during hysteresis.

2. The volume of a blob or a bubble maintains constant during the mobilization. In most experiments, the magnitude of capillary pressure acting on an air bubble is of $10^{2} \mathrm{~Pa}$ 
in a capillary with diameter of millimeters, while the hydrostatic pressure of the air bubble is 1 atm $\left(\approx 10^{5} \mathrm{~Pa}\right)$ (Srinivasan et al. 2013; Hsu and Hilpert 2016). Therefore, the compression of air bubbles caused by capillary pressure can be ignored in those experiments. Considering a reservoir with a depth over $1000 \mathrm{~m}$, the hydrostatic pressure may be over $10^{7} \mathrm{~Pa}$. The capillary pressure will increase to the same magnitude as the hydrostatic pressure when the diameter of pore space decreases to $10 \mathrm{~nm}$. Therefore, this assumption is still valid when the diameter of pores or throats is much larger than $10 \mathrm{~nm}$ (from hundreds of nanometers to micrometers). As for microscale capillaries, the assumption still holds.

3. During mobilization, if one interface starts moving, it moves at low capillary number and the contact angle remains to be the advancing or receding angle. This assumption is based on the velocity independence of contact angle at low capillary number $10^{-9}-10^{-5}$, supported by the experiments (Johnson et al. 1977; Morrow and Nguyen 1982; Lei et al. 2018);

4. The viscous effect on pressure drop can be ignored. Following the assumptions (1) and (3), the bulk fluid does not flow along the capillary during hysteresis. And even the bulk fluid flows as one interface starts moving, the viscous pressure can also be ignored compared with the capillary pressure

5. An equilibrium state is assumed for a blob or a bubble in the beginning of the mobilization. The initial value of contact angles at two interfaces is assumed to be $\theta_{e}$.

\subsubsection{Analysis of Capillary Pressure and Contact Angles}

The contact angle for the interface that is inclined to advance is denoted as $\theta^{\prime}$, and the other inclined to recede is denoted as $\theta^{\prime \prime}$. The initial values of contact angles are denoted as $\theta_{e}$, which satisfies $\theta_{\text {rec }}<\theta_{e}<\theta_{\text {adv }}$. As the inlet pressure $P_{i}$ slowly increases, $\theta^{\prime}$ will slowly rise from $\theta_{e}$ to $\theta_{\text {adv }}$, while $\theta^{\prime \prime}$ will slowly decrease from $\theta_{e}$ to $\theta_{\text {rec }}$ (Srinivasan et al. 2013, 2015). Before contact points start moving, the viscous pressure drop within bulk fluids can be ignored. Thus, pressures $\left(P_{1}, P_{2}, P_{3}\right.$ and $P_{4}$, as shown in Fig. 2) equal at two ends of a bulk fluid, as given by:

$$
P_{i}=P_{1}, \quad P_{2}=P_{3}, \quad P_{4}=P_{o}
$$

For a blob, pressure drops across two interfaces are given by:

$$
\left\{\begin{array}{c}
P_{1}-P_{2}=\frac{2 \gamma}{R} \cos \theta^{\prime \prime} \\
P_{3}-P_{4}=-\frac{2 \gamma}{R} \cos \theta^{\prime}
\end{array}\right.
$$

For a bubble, pressure drops at interfaces are:

Fig. 2 Definition of pressures and contact angles of a blob and bubble in a capillary

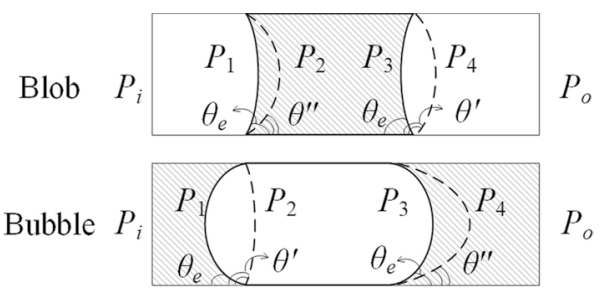




$$
\left\{\begin{array}{c}
P_{1}-P_{2}=-\frac{2 \gamma}{R} \cos \theta^{\prime} \\
P_{3}-P_{4}=\frac{2 \gamma}{R} \cos \theta^{\prime \prime}
\end{array}\right.
$$

Combining Eqs. (2), (3) and (4), we could have the total pressure drop, which has the same expression for a blob and a bubble.

$$
P_{i}-P_{o}=\frac{2 \gamma}{R}\left(\cos \theta^{\prime \prime}-\cos \theta^{\prime}\right)
$$

As demonstrated in Fig. 2, if the microtube is taken as a plane or a gap between two parallel plates, the volume change $v(\alpha, \beta)$ is solely induced by the change of contact angle from $\alpha$ to $\beta$ and is expressed by the difference of two circular segments. For a circular tube, the volume change $v(\alpha, \beta)$ is expressed by the difference of two spherical caps with contact angles from $\alpha$ to $\beta$.

Here, the expression of volume change in a circular tube is given in Eq (6), wherein $R$ denotes the radius of the circular tube. Detailed derivation of the volume change can be found in "Appendix 1."

$$
v(\alpha, \beta)=\left[\frac{(1-\sin \alpha)^{2}(2+\sin \alpha)}{\cos ^{3} \alpha}-\frac{(1-\sin \beta)^{2}(2+\sin \beta)}{\cos ^{3} \beta}\right] \frac{\pi R^{3}}{3}
$$

The two angles must satisfy $0 \leq \alpha \leq \beta \leq \pi$ to obtain a nonnegative value of $v(\alpha, \beta)$ (Fig. 3).

The variations of $\theta^{\prime}$ and $\theta^{\prime \prime}$ are linked up with each other, due to the conservation of the blob/bubble volume. One interface shrinks and the other enlarges with $\Delta V_{1}=\Delta V_{2}$, as illustrated in Fig. 4. The solid lines indicate the original interfaces, and the dashed lines indicate the interfaces when $\theta^{\prime}=\theta_{\text {adv }}$ and $\theta^{\prime \prime}=\theta_{\text {rec }}$

The advancing interface will finish the hysteresis process when $\theta^{\prime}=\theta_{\text {adv }}$, and the receding interface will do the same when $\theta^{\prime \prime}=\theta_{\text {rec }}$. Interestingly, $\theta^{\prime}$ and $\theta^{\prime \prime}$ do not necessarily reach $\theta_{\text {adv }}$ and $\theta_{\text {rec }}$ at the same time. Only when $v\left(\theta_{\text {rec }}, \theta_{e}\right)=v\left(\theta_{e}, \theta_{\text {adv }}\right)$, will the two interfaces start moving together. Otherwise, if $v\left(\theta_{\text {rec }}, \theta_{e}\right)<v\left(\theta_{e}, \theta_{\text {adv }}\right)$, the receding interface will have to slip a distance $L_{s}$ to even the change of volumes, where $\Delta V_{2}=v\left(\theta_{\text {rec }}, \theta_{e}\right)+\pi R^{2} L_{s}$ and $\Delta V_{1}=v\left(\theta_{e}, \theta_{\text {adv }}\right)$. And if $v\left(\theta_{\text {rec }}, \theta_{e}\right)>v\left(\theta_{e}, \theta_{\text {adv }}\right)$, the advancing interface will have to slip a distance $L_{s}$; then, we have $\Delta V_{2}=v\left(\theta_{\text {rec }}, \theta_{e}\right)$ and $\Delta V_{1}=v\left(\theta_{e}, \theta_{\text {adv }}\right)+\pi R^{2} L_{s}$. These situations are depicted in Fig. 4. Thus, before two interfaces move together, the slip distance $L_{s}$ of one interface can be expressed as:

$$
L_{s}=\frac{\left|v\left(\theta_{\mathrm{rec}}, \theta_{e}\right)-v\left(\theta_{e}, \theta_{\mathrm{adv}}\right)\right|}{\pi R^{2}}
$$

Fig. 3 Volume change $v(\alpha, \beta)$ is solely induced by contact angle variation from $\alpha$ to $\beta$

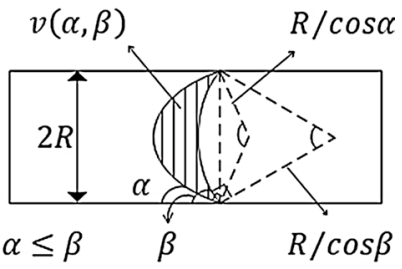




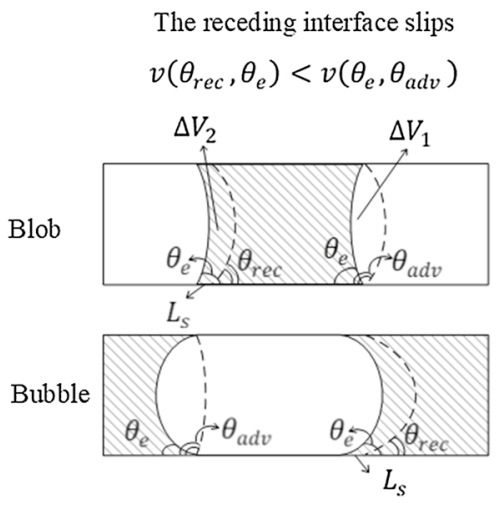

The advancing interface slips

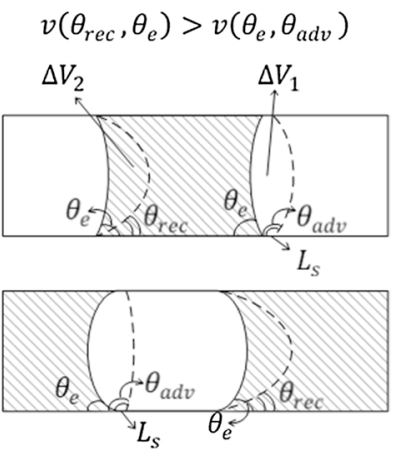

Fig. 4 When a blob or a bubble is mobilized, two contact angles at two interfaces will experience hysteresis at the same time. Solid lines indicate the interfaces at time zero, and the dashed lines indicate the interfaces on the verge of blob/bubble movement. Threshold angles may not be reached at the same time, and one interface might slip a certain distance $L_{s}$ to remain the constant volume of the blob or bubble

According to Eq. (7), the slip distance can be expressed as a dimensionless form as in Eq. (8), which is the ratio of slip distance to the radius of the circular tube:

$$
\begin{aligned}
\tilde{L}_{s}=\frac{L_{s}}{R} & =\frac{1}{3} \mid \frac{\left(1-\sin \theta_{\text {rec }}\right)^{2}\left(2+\sin \theta_{\text {rec }}\right)}{\cos ^{3} \theta_{\text {rec }}}+\frac{\left(1-\sin \theta_{\text {adv }}\right)^{2}\left(2+\sin \theta_{\text {adv }}\right)}{\cos ^{3} \theta_{\text {adv }}} \\
& -\frac{2\left(1-\sin \theta_{e}\right)^{2}\left(2+\sin \theta_{e}\right)}{\cos ^{3} \theta_{e}} \mid
\end{aligned}
$$

The relationship in Eq. (8) can be plotted in 3D surface, as given in Fig. 5. Under different $\theta_{e}, \theta_{\text {adv }}$ and $\theta_{\text {rec }}$ have to satisfy different relations to achieve zero slip distance $\tilde{L}_{s}$. The slip distance is closely related to contact angle hysteresis $(\mathrm{CAH})$. When $\mathrm{CAH}=0$, one has $\theta_{\text {adv }}=\theta_{\text {rec }}=\theta_{e}$, and obviously, the right side of Eq. (8) is zero and $\tilde{L}_{s}=0$.

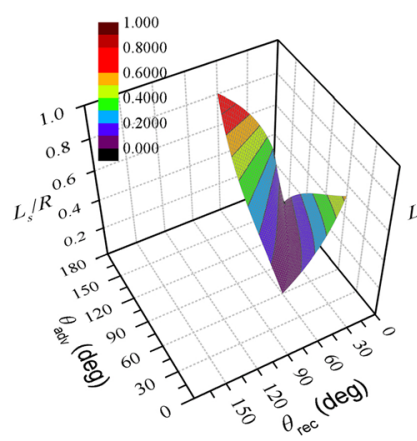

(a) $\theta_{e}=60^{\circ}$

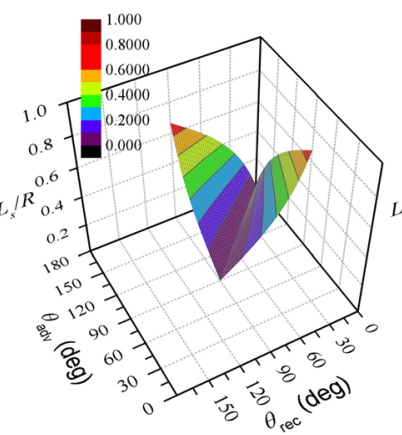

(b) $\theta_{e}=90^{\circ}$

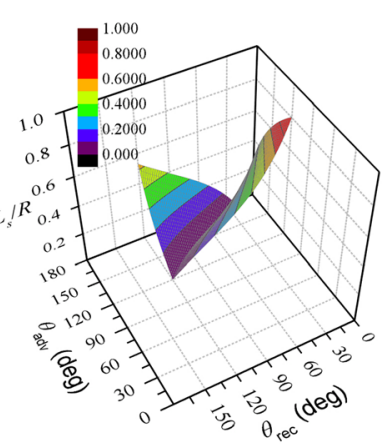

(c) $\theta_{e}=120^{\circ}$

Fig. 5 Distribution of dimensionless slip distance $\tilde{L}_{s}$ with different $\theta_{e}$ 


\subsubsection{Coordinated Variation of Contact Angles}

Before neither $\theta^{\prime}$ nor $\theta^{\prime \prime}$ reaches threshold angles, the volume change at two interfaces is equal, which leads to an implicit function of $\theta^{\prime}, \theta^{\prime \prime}$ and $\theta_{e}$ in Eq. (9) (take circular tube as an example).

$$
\begin{aligned}
& v\left(\theta^{\prime \prime}, \theta_{e}\right)=v\left(\theta_{e}, \theta^{\prime}\right) \\
& \Rightarrow \quad \frac{\left(1-\sin \theta^{\prime}\right)^{2}\left(2+\sin \theta^{\prime}\right)}{\cos ^{3} \theta^{\prime}}+\frac{\left(1-\sin \theta^{\prime \prime}\right)^{2}\left(2+\sin \theta^{\prime \prime}\right)}{\cos ^{3} \theta^{\prime \prime}}=\frac{2\left(1-\sin \theta_{e}\right)^{2}\left(2+\sin \theta_{e}\right)}{\cos ^{3} \theta_{e}}
\end{aligned}
$$

$\theta^{\prime}$ and $\theta^{\prime \prime}$ can be determined by Eqs. (5) and (9), once the pressure drop $P_{i}-P_{o}$ and the equilibrium angle $\theta_{e}$ are given.

Figure $6 \mathrm{a}, \mathrm{b}$ demonstrates the coordinated variation between $\theta^{\prime}$ and $\theta^{\prime \prime}$ under situations of different $\theta_{e}$. When $\theta_{e}<90^{\circ}$, for a large change of $\theta^{\prime}$, there will be a small change in $\theta^{\prime \prime}$; when $\theta_{e}>90^{\circ}$, for a small change of $\theta^{\prime}$, there will be a large change in $\theta^{\prime \prime}$. Only when $\theta_{e}=90^{\circ}, \theta^{\prime}$ and $\theta^{\prime \prime}$ will change equally. If $\theta_{e}=90^{\circ}, \theta^{\prime}$ and $\theta^{\prime \prime}$ will start off at $\theta^{\prime}=\theta^{\prime \prime}=90^{\circ}$ and follow the trajectory predicted by the implicit function $v\left(\theta^{\prime \prime}, 90^{\circ}\right)=v\left(90^{\circ}, \theta^{\prime}\right)$ which is line $A-B$ in Fig. 6b. If threshold angles $\left(\theta_{\text {adv }}, \theta_{\text {rec }}\right)$ are located at point $C$, then variations will go down through line $B-C$, which is parallel to the $Y$-axis. Similarly, variations will go straight through line $B-D$ parallel to the $x$-axis, if threshold angles are located at point $D$. In line $B-C, \theta^{\prime \prime}$ keeps decreasing while $\theta^{\prime}=\theta_{\text {adv }}$ meaning the advancing interface is slipping, corresponding to the situation of $v\left(\theta_{\text {rec }}, \theta_{e}\right)>v\left(\theta_{e}, \theta_{\text {adv }}\right)$. In line $B-D$, the receding interface will slip with $\theta^{\prime \prime}=\theta_{\text {rec }}$ while $\theta^{\prime}$ keeps increasing, corresponding to the situation of $v\left(\theta_{\text {rec }}, \theta_{e}\right)<v\left(\theta_{e}, \theta_{\text {adv }}\right)$. Therefore,

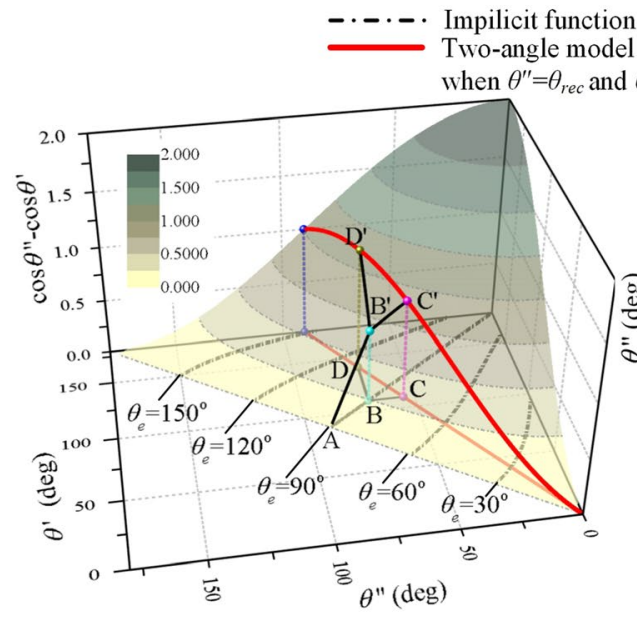

(a)

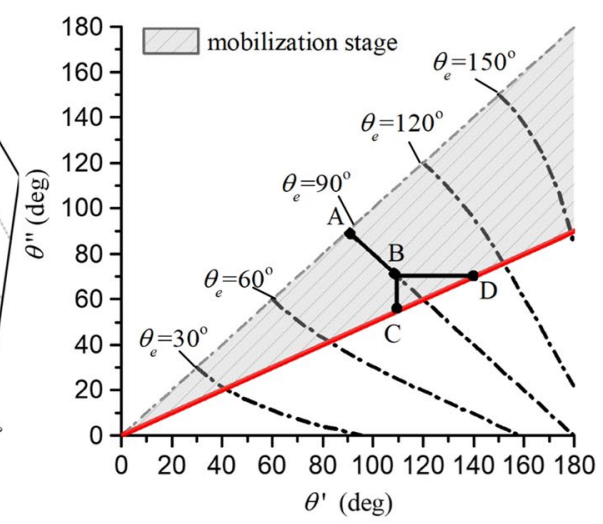

(b)

Fig. 6 Variation of two angles of a bubble or blob during. Starting from $\theta_{e}, \theta^{\prime}$ increases and $\theta^{\prime \prime}$ decreases, which obeys the implicit function $v\left(\theta^{\prime \prime}, \theta_{e}\right)=v\left(\theta_{e}, \theta^{\prime}\right)$. Trajectory $A-B-C$ demonstrates an example of variation of $\theta^{\prime}$ and $\theta^{\prime \prime}$ when $\theta^{\prime}$ reaches $\theta_{\text {adv }}$ first; trajectory $A-B-D$ demonstrates the situation where $\theta^{\prime \prime}$ reaches $\theta_{\text {rec }}$ first. Trajectories $A-B^{\prime}-C^{\prime}$ and $A-B^{\prime}-D^{\prime}$ are projections of $A-B-C$ or $A-B-D$, and they both climbing up the surface of $\cos \theta^{\prime \prime}-\cos \theta^{\prime}$, indicating that the capillary pressure is rising during the process 
according to the location of threshold angles $\left(\theta_{\mathrm{adv}}, \theta_{\text {rec }}\right)$, the mobilization of a blob/bubble will finish, through trajectories $A-B-C$ or $A-B-D$ on the $\theta^{\prime}-\theta^{\prime \prime}$ plane.

Figure $6 \mathrm{a}$ also shows the $3 \mathrm{D}$ surface of $\cos \theta^{\prime \prime}-\cos \theta^{\prime}$, which reflects the magnitude of pressure drop $P_{i}-P_{o}$ as in Eq. (5). Trajectories $A-B^{\prime}-C^{\prime}$ and $A-B^{\prime}-D^{\prime}$ are projections of $A-B-C$ or $A-B-D$ on the surface of $\cos \theta^{\prime \prime}-\cos \theta^{\prime}$. It is obvious that both trajectories $A-B^{\prime}-C^{\prime}$ and $A-B^{\prime}-D^{\prime}$ are climbing up the surface, meaning that $\cos \theta^{\prime \prime}-\cos \theta^{\prime}$ rises as the two angles go through $A-B-C$ or $A-B-D$. Equations (5) and (9) together describe how the two angles vary during mobilization stage.

To predict the ending of the mobilization stage, the knowledge of $\theta_{\text {adv }} / \theta_{\text {rec }}$ relationship is needed. A previous study proposed an empirical relationship between $\theta_{\text {adv }}$ and $\theta_{\text {rec }}$ based on different experiments (Lei et al. 2018). These experiments measure the advancing and receding angles of different liquids on the same solid surface. And in some experiments, the solid surface is carefully modified to achieve desired roughness. A linear trend between $\theta_{\text {adv }}$ and $\theta_{\text {rec }}$ is found and given by (Lei et al. 2018):

$$
\theta_{\text {rec }}=(1-k) \theta_{\mathrm{adv}}
$$

The factor $k(0<k<1)$ is found to be positively related to the roughness of solid surface (Lei et al. 2018).

This linear relationship can be used to mark the area of mobilization stage. The linear trend is plotted in Fig. $6 \mathrm{~b}$ as red lines, including a projection on the surface of $\cos \theta^{\prime \prime}-\cos \theta^{\prime}$. The area of mobilization stage is filled with color gray and slashes. Points $C$ and $D$ are assumed to satisfy $\theta_{\text {rec }}=(1-k) \theta_{\text {adv }}$, so they are plotted on the red lines. Starting from the equilibrium angle $\theta_{e}, \theta^{\prime}$ and $\theta^{\prime \prime}$ will finally reach threshold angles $\left(\theta_{\text {adv }}, \theta_{\text {rec }}\right)$ located at line $\theta_{\text {rec }}=(1-k) \theta_{\text {adv }}$. The area surrounded by $\theta^{\prime}=\theta^{\prime \prime}$ and $\theta_{\text {rec }}=(1-k) \theta_{\text {adv }}$ is where the mobilization may occur. Except for marking the possible area of mobilization stage, the $\theta_{\text {adv }} / \theta_{\text {rec }}$ relationship can help predict the maximum blob/bubble mobilization pressure for a given solid surface.

\subsubsection{Maximum Mobilization Pressure on a Given Solid Surface}

After the movement of a blob/bubble has been initiated, the contact angles remain to be $\theta_{\text {adv }}$ and $\theta_{\text {rec }}$, respectively, for the advancing and receding interfaces with $C a<10^{-5}$. There are six scenarios of blob or bubble movement according to categories of $\theta_{\text {adv }} / \theta_{\text {rec }}$ relationship, as depicted in Fig. 7a.

For category (1) where $\theta_{\text {adv }}$ and $\theta_{\text {rec }}$ are both over $\pi / 2$, the capillary pressure at the receding interface acts as a driving force while the one at the advancing interface as a resisting force for the movement. Because $\pi / 2<\theta_{\text {rec }}<\theta_{\text {adv }}<\pi$, the resistance is always larger than the propulsion $2 \gamma\left|\cos \theta_{\text {adv }}\right| / R>2 \gamma\left|\cos \theta_{\text {rec }}\right| / R$. Thus, either blobs or bubbles in category (1) possess a mobilization pressure $P_{m}=2 \gamma\left(\left|\cos \theta_{\text {adv }}\right|-\left|\cos \theta_{\text {rec }}\right|\right) / R$. But noted that $\left|\cos \theta_{\text {adv }}\right|=-\cos \theta_{\text {adv }}$ and $\left|\cos \theta_{\text {rec }}\right|=-\cos \theta_{\text {rec }}$ when $\pi / 2<\theta_{\text {rec }}<\theta_{\text {adv }}<\pi$. So, the mobilization pressure can be expressed as $P_{m}=2 \gamma\left(\cos \theta_{\text {rec }}-\cos \theta_{\text {adv }}\right) / R$. A classic example of this category is a water droplet on lotus leaves, where the advancing angle reaches $160^{\circ}$ and the receding angle is $150^{\circ}$ (Quéré 2008).

For category (2) where $\theta_{\mathrm{adv}}>\pi / 2, \theta_{\mathrm{rec}}<\pi / 2$, the capillary pressure at two interfaces is both resisting the movement. So, the mobilization pressure is $P_{m}=2 \gamma\left(\left|\cos \theta_{\text {adv }}\right|+\left|\cos \theta_{\text {rec }}\right|\right) / R$. Since $0<\theta_{\text {rec }}<\pi / 2<\theta_{\text {adv }}<\pi$, we have $\left|\cos \theta_{\text {adv }}\right|=-\cos \theta_{\text {adv }}, \quad\left|\cos \theta_{\text {rec }}\right|=\cos \theta_{\text {rec }} \quad$ and $\quad P_{m}=2 \gamma\left(\cos \theta_{\text {rec }}-\cos \theta_{\text {adv }}\right) / R$. An 


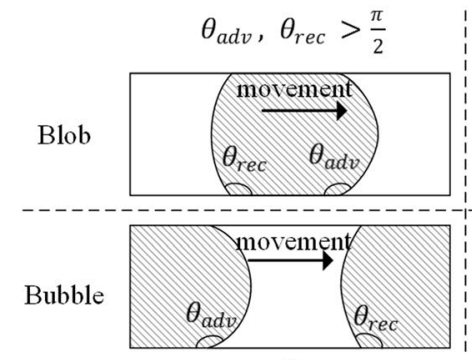

(i)
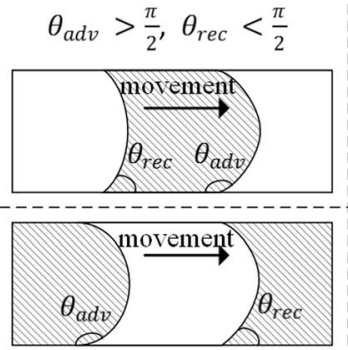

(ii)

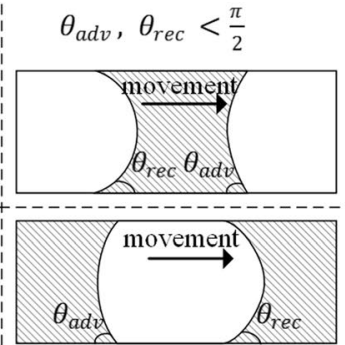

(iii)

(a)

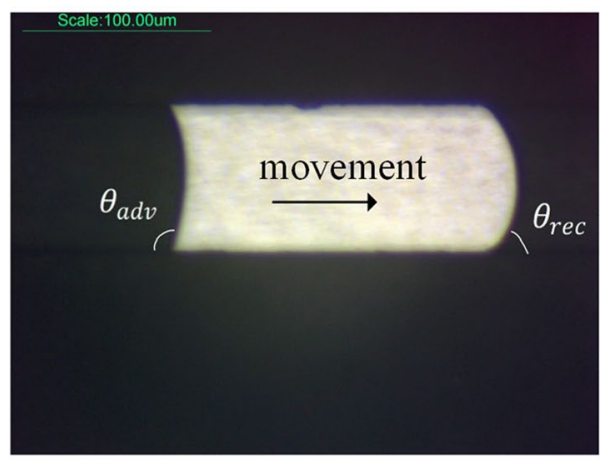

(b)

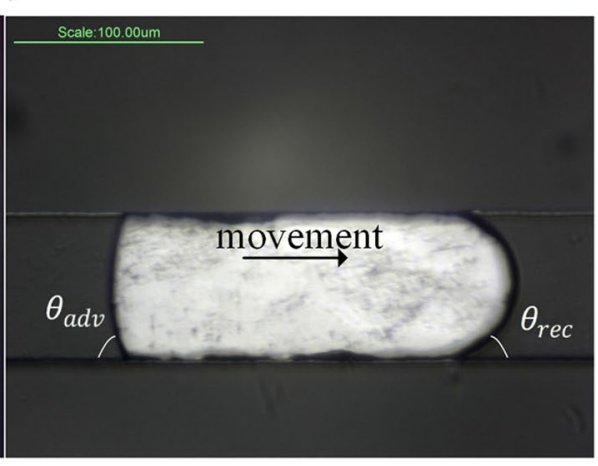

(c)

Fig. 7 Different scenarios of blob and bubble movement: a A blob/bubble has two interfaces, one of which advances and the other recedes at the same time. There are six scenarios in total; $\mathbf{b}$ the movement of a bubble trapped by $20 \mathrm{wt} \%$ glucose solution in an 80 - $\mu \mathrm{m}$-wide rectangular capillary; c the movement of a bubble trapped by PEG200 in an $80-\mu \mathrm{m}$-wide rectangular capillary

example of this category is demonstrated in Fig. $6 \mathrm{~b}$, where an air bubble is trapped by glucose solution in an $80 \times 20$ (width $\times$ depth) $\mu$ m capillary.

For category (3) where $\theta_{\mathrm{adv}}$ and $\theta_{\mathrm{rec}}$ are both less than $\pi / 2$, the advancing interface induces a capillary pressure that drives the movement, but the receding interface carries a capillary pressure resisting the movement. Thus, the mobilization pressure is $P_{m}=2 \gamma\left(\left|\cos \theta_{\text {rec }}\right|-\left|\cos \theta_{\text {adv }}\right|\right) / R$, which can be reduced as $P_{m}=2 \gamma\left(\cos \theta_{\text {rec }}-\cos \theta_{\text {adv }}\right) / R$, since $\left|\cos \theta_{\text {adv }}\right|=\cos \theta_{\text {adv }}, \quad\left|\cos \theta_{\text {rec }}\right|=\cos \theta_{\text {rec }} \quad$ for $0<\theta_{\text {rec }}<\theta_{\text {adv }}<\pi / 2$. An example of this category is demonstrated in Fig. 6c, where an air bubble is trapped by polyethylene 200 (PEG200) in an $80 \times 20$ (width $\times$ depth) $\mu \mathrm{m}$ capillary.

For all three categories, the mobilization pressure of multiple blobs/bubbles can be easily added up if they are within the same capillary. The mobilization pressure for $n$ blobs or bubbles is given by:

$$
P_{m}=\frac{2 \gamma n}{R}\left(\cos \theta_{\text {rec }}-\cos \theta_{\text {adv }}\right)
$$

Equation (11) describes the mobilization pressure for different systems of wettability, and it is in agreement with the formula mentioned by Smith and Crane (1930). 
To predict the maximum mobilization pressure according to Eq. (11), the knowledge of $\theta_{\text {adv }} / \theta_{\text {rec }}$ relationship is needed. Once again, the linear trend between $\theta_{\text {adv }}$ and $\theta_{\text {rec }}$ in Eq. (10) is incorporated to predict the maximum mobilization pressure.

Firstly, two sides of Eq. (11) are multiplied by $R /(2 \gamma n)$ to obtain a dimensionless mobilization $\tilde{P}_{m}$ :

$$
\tilde{P}_{m}=\cos \theta_{\text {rec }}-\cos \theta_{\text {adv }}
$$

Incorporating Eq. (12) with Eq. (10), $\theta_{\text {rec }}$ can be eliminated in Eq. (12)

$$
\tilde{P}_{m}=\cos \left[(1-k) \theta_{\mathrm{adv}}\right]-\cos \theta_{\mathrm{adv}}
$$

$\tilde{P}_{m}$ obtains its maximum value on a given solid surface ( $k$ is given) when its derivative is zero.

$$
\frac{\partial \tilde{P}_{m}}{\partial \theta_{\text {adv }}}=-(1-k) \sin \left[(1-k) \theta_{\text {adv }}\right]+\sin \theta_{\text {adv }}
$$

However, no explicit results can be derived from equating Eq. (14) with zero.

An approximation by quadratic function is proposed to replace the derivative of $\tilde{P}_{m}$ within the range of $0 \leq \theta_{\mathrm{adv}} \leq \pi$, given by Eq. (15). Detailed derivation of the approximation is given in "Appendix 2."

$$
\left(\frac{\partial \tilde{P}_{m}}{\partial \theta_{\text {adv }}}\right)_{\text {approx. }}=\frac{4}{\pi} \theta_{\text {adv }}\left[\frac{(1-k)^{3}-1}{\pi} \theta_{\text {adv }}-(1-k)^{2}+1\right]
$$

Equating Eq. (15) with zero, the $\theta_{\text {adv }}$ leading to maximum $\tilde{P}_{m}$ can be obtained.

$$
\theta_{\mathrm{adv}}=\pi \frac{(1-k)^{2}-1}{(1-k)^{3}-1}
$$

Substituting Eq. (16) in Eq. (13) gives the maximum mobilization pressure (approximation) $\tilde{P}_{m}$.

$$
\tilde{P}_{m, \max } \approx \cos \left[\pi \frac{(1-k)^{3}-(1-k)}{(1-k)^{3}-1}\right]-\cos \left[\pi \frac{(1-k)^{2}-1}{(1-k)^{3}-1}\right]
$$

The comparison between the original derivative Eq. (14) and the approximation function Eq. (15) is demonstrated in Fig. 8. The maximum values of mobilization pressure are obtained by both numerical methods with MATLAB codes and the approximation Eq. (17). As shown in Fig. 8, the relative error of the approximation is well below $0.35 \%$, which is acceptable for predicting the maximum mobilization pressure. When $k=0$, i.e., no contact angle hysteresis, the maximum mobilization pressure is zero. However, as $k$ rises to $1, \tilde{P}_{m}$ increases from 0 to 2 . Since $k$ is positively correlated with surface roughness, we could expect that rough surfaces possess larger $\tilde{P}_{m}$. 
Fig. 8 Maximum mobilization pressures obtained by numerical method (the red line) and approximation Eq. (17) (red squares). Relative errors between them are less than $0.35 \%$, indicating that the approximation provides accurate predictions

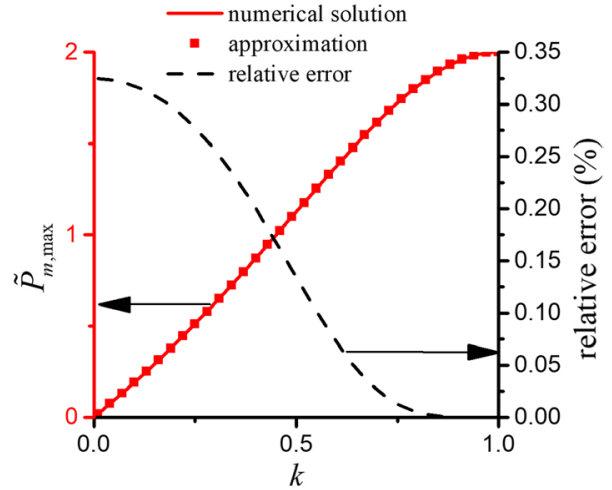

\section{Comparison with Experiments and Discussions}

Srinivasan et al. recorded the variation of two angles of a liquid slug in a dry tube with diameter of $1.5 \mathrm{~mm}$, as plotted in Fig. 9a and marked as \#1 and \#2 (Srinivasan et al. 2013). The continuous measurement of contact angles was carried out during the mobilization stage (i.e., before the slug starts moving). In another experiment, Srinivasan et al. only measured contact angles at the beginning and the end of the mobilization and obtained two points for different liquids in the same tube, marked as \#3 and \#4 in Fig. 9a (Srinivasan et al. 2015). The contact angle at the interface that is about to advance is denoted as $\theta^{\prime}$, and the contact angle at the interface that is about to recede is denoted as $\theta^{\prime \prime}$. Symbols of different colors represent the measured points from experiments, and curves of different colors are the corresponding fitting results computed by the implicit function of $\theta^{\prime}$ and $\theta^{\prime \prime}$ in Eq. (9). The equilibrium contact angles are computed by averaging the closest values of $\theta^{\prime}$ and $\theta^{\prime \prime}$ for experiments \#1 and \#2, which gives $\theta_{e}=53.9^{\circ}$ and $\theta_{e}=52.7^{\circ}$, respectively. For experiments \#3 and \#4, contact angles are measured in the beginning and the end of

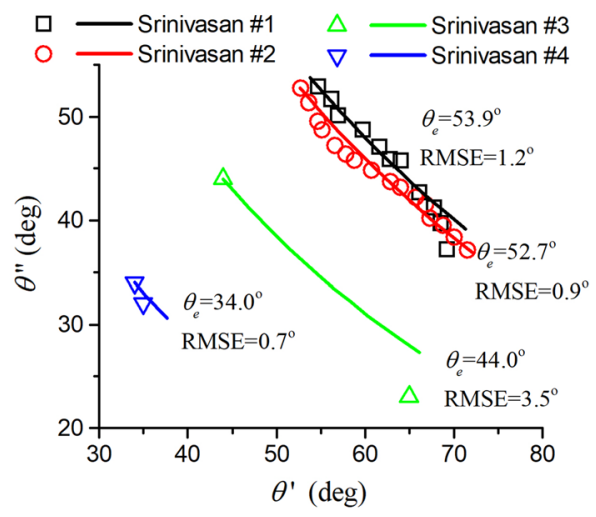

(a)

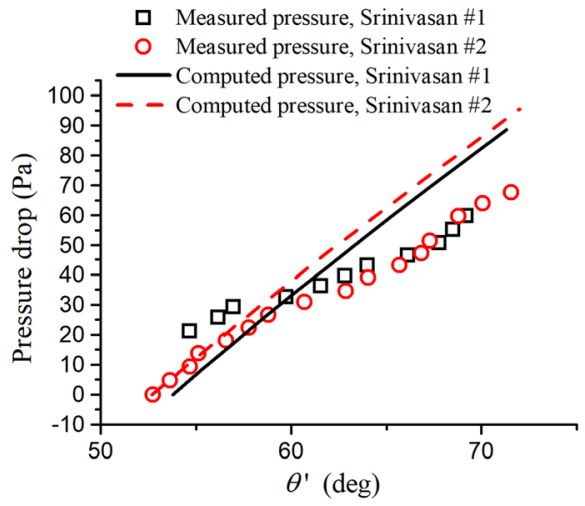

(b)

Fig. 9 a Variation of $\theta^{\prime}$ and $\theta^{\prime \prime}$ during the mobilization stage of a liquid slug in a 1.5-mm-diameter tube. Symbols represent the measured data, and solid lines are computed by the implicit function $v\left(\theta^{\prime \prime}, \theta_{e}\right)=v\left(\theta_{e}, \theta^{\prime}\right)$, Eq. (13). b: The pressure increases during the variation of $\theta^{\prime}$ and $\theta^{\prime \prime}$. The solid line and the dashed line are computed results; symbols are measured data 
mobilization, and there are only two points under the symbol of Srinivasan \#3 and Srinivasan \#4. $\theta_{e}$ is the contact angle measured at the beginning of mobilization. All four experiments adequately follow the trend of the implicit function $v\left(\theta^{\prime \prime}, \theta_{e}\right)=v\left(\theta_{e}, \theta^{\prime}\right)$, and the maximum root-mean-square error (RMSE) is $2.9^{\circ}$ for experiment \#3.

As the liquid slug is driven by constant flow rate injection, $\theta^{\prime}$ and $\theta^{\prime \prime}$ continuously vary and the capillary pressure is found to be rising during the process (Srinivasan et al. 2013, 2015). Figure $9 \mathrm{~b}$ demonstrates the measured data of pressure drop induced by mobilizing the liquid slug in the experiments of Srinivasan et al. (Srinivasan et al. 2013). The symbols in Fig. $9 \mathrm{~b}$ are the computational results of capillary pressure. Wherein, $\theta^{\prime}$ and $\theta^{\prime \prime}$ are calculated by the implicit function Eq. (9), and the pressure drop is computed by Eq. (5). The surface tension of water $\gamma=73 \mathrm{mN} / \mathrm{m}$ and the tube radius $R=0.75 \mathrm{~mm}$ are directly taken from Srinivasan et al. (2013). Measured data basically follow the trend of theoretical computation. However, the deviation is obvious and might be caused by the pressure measurement, which is conducted in low pressure range (less than $100 \mathrm{~Pa}$ ) and is easily influenced by system uncertainties.

The linear relationship between $\theta_{\text {adv }}$ and $\theta_{\text {rec }}$ is used in this work to predict the boundary of the mobilization stage of blobs/bubbles. The factor $k$ characterizes the area of the mobilization stage and also indicates the maximum mobilization pressure that could exist on a given solid surface. There are experiments measuring the advancing and receding angles of different liquids on the same solid which enables the estimation of $k$. Figure 10 depicts the relationship of $\theta_{\text {adv }}$ versus $\cos \theta_{\text {rec }}-\cos \theta_{\text {adv }}$, and the symbols are plotted according to the measured data of $\theta_{\mathrm{adv}}$ and $\theta_{\text {rec }}$ from Ramos and Tanguy (2006), Meiron et al. (2004) and Morrow and Nguyen (1982). Solid lines in Fig. 10 are computed by Eq. (13) that replaces $\theta_{\text {rec }}$ with $(1-k) \theta_{\text {adv }}$. The fitting results of $k$ for the experiments aforementioned are given in Lei et al. (2018). The locations of stars in Fig. 10 are computed by Eqs. (16) and (17), which are approximations for the maximum mobilization pressure. All experiments well follow the trend of Eq. (13), i.e., well predicted by the linear $\theta_{\text {adv }} / \theta_{\text {rec }}$ relationship. But most experiments do not measure the advancing and receding angles that will lead to the maximum mobilization pressure. Equations (16) and (17) provide a way to predict the maximum mobilization pressure that could possibly exist on a given solid surface (with a given $k$ ). As seen from Fig. 10, the maximum mobilization pressure and the $\theta_{\text {adv }}$ where the maximum pressure occurs are raised as the $k$ increases. As the positive correlation between $k$ and surface roughness has been found (Lei et al. 2018), rougher surfaces possess larger mobilization pressure under the same $\theta_{\mathrm{adv}}$, as well

Fig. 10 Advancing angle $\theta_{\text {adv }}$ versus dimensionless mobilization pressure $\cos \theta_{\text {rec }}-\cos \theta_{\text {adv }}$. Solid lines are computed by Eq. (13), wherein $k$ s are given in Lei et al. (2018). Star-shaped symbols are the maximum mobilization pressure under different $k$. Other symbols are plotted according to the measured data of $\theta_{\mathrm{adv}}$ and $\theta_{\text {rec }}$ in different experiments

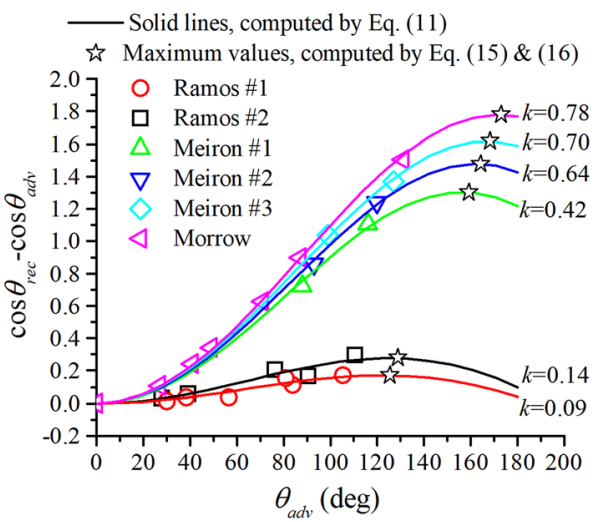

Springer 
as larger maximum mobilization pressure. If the mobilization pressure is expected to be as small as possible, for a given solid surface, liquid with small contact angle hysteresis (CAH) would serve the purpose better. According to the correlation $\theta_{\mathrm{rec}}=(1-k) \theta_{\mathrm{adv}}$, small CAH indicates that the liquid possesses small $\theta_{\text {adv }}$ on the given solid surface. In all, the liquid-solid system with small $k$ and small $\theta_{\text {adv }}$ would be beneficial to obtain small mobilization pressure.

\section{Conclusion}

Based on the behavior of a single liquid-gas interface, a model is proposed to quantitatively describe the coordinated variation of contact angles of double interfaces during mobilization. The capillary pressure of a blob/bubble and the contact angles can be fully obtained through the model.

The variation of contact angles of the advancing and receding interfaces can be computed by the implicit function Eq. (9), which is in good agreement with experiments (Srinivasan et al. 2013, 2015). Other experiments support the conclusion that the pressure drop during mobilization keeps rising when $\theta^{\prime}$ and $\theta^{\prime \prime}$ continuously vary until they reach threshold angles $\theta_{\text {adv }}$ and $\theta_{\text {rec }}$, which is predicted by Eqs. (5) and (9) together (Srinivasan et al. 2013, 2015; Uchiyama et al. 2015). When $\theta_{e}<90^{\circ}$, for a large change of $\theta^{\prime}$ there will be a small change in $\theta^{\prime \prime}$; when $\theta_{e}>90^{\circ}$, for a small change of $\theta^{\prime}$ there will be a large change in $\theta^{\prime \prime}$. Only when $\theta_{e}=90^{\circ}, \theta^{\prime}$ and $\theta^{\prime \prime}$ will change equally. The mobilization pressure of bubbles/blobs has long been recognized as a result of contact angle hysteresis (Smith and Crane 1930; Hsu and Hilpert 2016; Srinivasan et al. 2015), and the model proposed in this work provides an insight into how the two contact angles experience hysteresis to maintain the constant volume of a bubble/ blob during mobilization.

Two interfaces appearing in the tube simultaneously, their capillary pressure together baffle the mobilization or movement of a bubble or a blob. The scope of mobilization stage under different equilibrium angle $\theta_{e}$ can be specified by a two-angle model proposed in a previous study (Lei et al. 2018). According to the two-angle model, the maximum mobilization pressure of a blob/bubble can be obtained by a quadratic approximation. The dimensionless mobilization pressure $\tilde{P}_{m}$ on a given solid surface will rise to a maximum before falling as the increase in the advancing angle $\theta_{\text {adv }}$. This trend is predicted by Eq. (13), and different experiments provide data on the rising part of $\tilde{P}_{m}$ (Morrow and Nguyen 1982; Ramos et al. 2003; Meiron et al. 2004). A rougher surface (with a larger $k$ ) will possess a larger maximum mobilization pressure and also a larger $\theta_{\text {adv }}$ where exists the maximum mobilization pressure. It has been found that the two-angle model can be used to predict the scope of mobilization stage and the maximum mobilization pressure on a given solid surface.

Acknowledgements This work was supported by the National Natural Science Foundation of China (Grant No. 41690132), the Strategic Priority Research Program of the Chinese Academy of Sciences (Grant No. XDA14010304), the National Natural Science Foundation of China (Grant No. 41574129) and the Major National Science and Technology Special Program of China (Grant No. 2017ZX05037-001).

\section{Appendix 1: The Volume Change Induced by Interface Deformation}

As for the volume change in a circular tube, one has to calculate the volume of a spherical cap $v_{\mathrm{SC}}$ first, depicted in Fig. 11. $v_{\mathrm{SC}}$ is given by: 


$$
v_{\mathrm{SC}}=\left|\frac{(1-\sin \alpha)^{2}(2+\sin \alpha)}{\cos ^{3} \alpha}\right| \frac{\pi R^{3}}{3}
$$

However, Eq. (18) has been rearranged to calculate $v_{\mathrm{SC}}$ as $\alpha$ approaches $\pi / 2$. Noting that $x \in[0, \pi]$, one has:

$$
\lim _{x \rightarrow \pi / 2} \frac{(1-\sin \alpha)^{2}(2+\sin \alpha)}{\cos ^{3} \alpha}=\left\{\begin{array}{c}
\lim _{x \rightarrow \pi / 2^{+}} \frac{(1-\sin \alpha)^{1 / 2}(2+\sin \alpha)}{(1+\sin \alpha)^{3 / 2}}=0 \\
\lim _{x \rightarrow \pi / 2^{-}}-\frac{(1-\sin \alpha)^{1 / 2}(2+\sin \alpha)}{(1+\sin \alpha)^{3 / 2}}=0
\end{array}\right.
$$

Thus, Eq. (19) is continuous within the range of $[0, \pi]$. If the contact angle changes from $\alpha$ to $\beta$, then the volume change in a circular tube is given by:

$$
v(\alpha, \beta)=\left[\frac{(1-\sin \alpha)^{2}(2+\sin \alpha)}{\cos ^{3} \alpha}-\frac{(1-\sin \beta)^{2}(2+\sin \beta)}{\cos ^{3} \beta}\right] \frac{\pi R^{3}}{3}
$$

\section{Appendix 2: The Quadratic Approximation}

We used a quadratic function $f_{t}(x)=\beta_{1}\left(x-\beta_{2}\right)\left(x-\beta_{3}\right)$ to approximate a sine function $f_{o}(x)=\alpha_{1} \sin \left(\alpha_{2} x\right)$ with $0 \leq x \leq \pi$, wherein $\beta_{1}, \beta_{2}$ and $\beta_{3}$ are undetermined parameters and $\alpha_{1}$ and $\alpha_{2}\left(0 \leq \alpha_{2}<1\right)$ are known parameters. $\beta_{1}, \beta_{2}$ and $\beta_{3}$ need to be expressed as functions of $\alpha_{1}$ and $\alpha_{2}$ for approximation. Within the range of $0 \leq x \leq \pi \Rightarrow 0 \leq \alpha_{2} x<\pi$, the curve of $f_{o}(x)=\alpha_{1} \sin \left(\alpha_{2} x\right)$ resembles a downward parabola, which can be expressed by $f_{t}(x)=\beta_{1}\left(x-\beta_{2}\right)\left(x-\beta_{2}\right)$. At least three equations (three pairs of coordinates) are needed to determine $\beta_{1}, \beta_{2}$ and $\beta_{3}$. For $f_{o}(x)=\alpha_{1} \sin \left(\alpha_{2} x\right)$, three equations are already fixed when $x=0, \alpha_{2} x=\pi / 2$ and $\alpha_{2} x=\pi$, and they are given by:

$$
\left\{\begin{array}{l}
f_{o}(0)=0 \\
f_{o}\left(\frac{\pi}{2 \alpha_{2}}\right)=\alpha_{1} \\
f_{o}\left(\frac{\pi}{\alpha_{2}}\right)=0
\end{array}\right.
$$

These three equations are used to determine the unknown parameters:

Fig. 11 Cross section of a circular tube, an interface and the contact angle $\alpha$

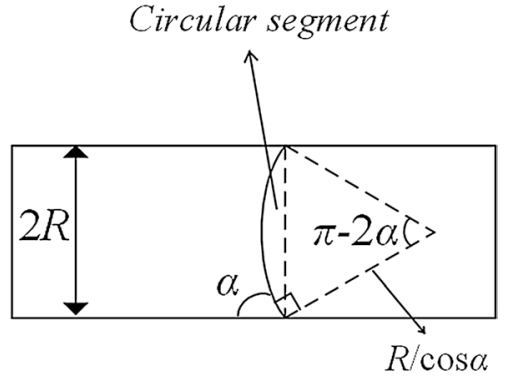




$$
\left\{\begin{array} { l } 
{ f _ { t } ( 0 ) = f _ { o } ( 0 ) } \\
{ f _ { t } ( \frac { \pi } { 2 \alpha _ { 2 } } ) = f _ { o } ( \frac { \pi } { 2 \alpha _ { 2 } } ) } \\
{ f _ { t } ( \frac { \pi } { \alpha _ { 2 } } ) = f _ { o } ( \frac { \pi } { \alpha _ { 2 } } ) }
\end{array} \Rightarrow \left\{\begin{array}{l}
\beta_{1} \beta_{2} \beta_{3}=0 \\
\beta_{1}\left(\frac{\pi}{2 \alpha_{2}}-\beta_{2}\right)\left(\frac{\pi}{2 \alpha_{2}}-\beta_{3}\right)=\alpha_{1} \\
\beta_{1}\left(\frac{\pi}{\alpha_{2}}-\beta_{2}\right)\left(\frac{\pi}{\alpha_{2}}-\beta_{3}\right)=0
\end{array}\right.\right.
$$

Solving the above equations, we will have:

$$
\left\{\begin{array} { l } 
{ \beta _ { 1 } = \frac { - 4 \alpha _ { 1 } \alpha _ { 2 } ^ { 2 } } { \pi ^ { 2 } } } \\
{ \beta _ { 2 } = 0 } \\
{ \beta _ { 3 } = \frac { \pi } { \alpha _ { 2 } } }
\end{array} \quad \text { or } \left\{\begin{array}{l}
\beta_{1}=\frac{-4 \alpha_{1} \alpha_{2}^{2}}{\pi^{2}} \\
\beta_{2}=\frac{\pi}{\alpha_{2}} \\
\beta_{3}=0
\end{array}\right.\right.
$$

Thus, to approximate the derivative of dimensionless mobilization pressure $\tilde{P}_{m}$, substituting the above results in the derivative, we obtain:

$$
\begin{aligned}
\frac{\partial \tilde{P}_{m}}{\partial \theta_{\mathrm{adv}}} & =-(1-k) \sin \left[(1-k) \theta_{\mathrm{adv}}\right]+\sin \theta_{\mathrm{adv}} \\
& \approx \frac{4(1-k)^{3}}{\pi^{2}} \theta_{\mathrm{adv}}\left(\theta_{\mathrm{adv}}-\frac{\pi}{1-k}\right)-\frac{4}{\pi^{2}} \theta_{\mathrm{adv}}\left(\theta_{\mathrm{adv}}-\pi\right)
\end{aligned}
$$

Rearranging Eq. (24), we will obtain the form of Eq. (15).

\section{References}

Bonn, D., Eggers, J., Indekeu, J., Meunier, J., Rolley, E.: Wetting and spreading. Rev. Mod. Phys. 81(2), 739-805 (2009). https://doi.org/10.1103/RevModPhys.81.739

Bora, R., Maini, B.B., Chakma, A.: Flow visualization studies of solution gas drive process in heavy oil reservoirs with a glass micromodel. SPE Reserv. Eval. Eng. 3(3), 224-229 (2000)

Chen, H., Amirfazli, A., Tang, T.: Modeling liquid bridge between surfaces with contact angle hysteresis. Langmuir 29(10), 3310-3319 (2013). https://doi.org/10.1021/la304870h

Gardescu, I.I.: Behavior of gas bubbles in capillary spaces. Trans. Am. Inst. Min. Met. Eng. 86, 351-370 (1930). https://doi.org/10.2118/930351-G

Hsu, S.Y., Glantz, R., Hilpert, M.: Pore-scale analysis of the effects of contact angle hysteresis on blob mobilisation in a pore doublet. Int. J. Oil Gas Coal Technol. 5(2/3), 207 (2012). https://doi.org/10.1504/ ijogct.2012.046321

Hsu, S.Y., Hilpert, M.: Pore-scale visualization of the mobilization of a partially wetting droplet. Adv. Water Resour. 95, 235-245 (2016). https://doi.org/10.1016/j.advwatres.2015.08.004

Jamali, M., Moghadam, A., Tafreshi, H.V., Pourdeyhimi, B.: Droplet adhesion to hydrophobic fibrous surfaces. Appl. Surf. Sci. 456, 626-636 (2018). https://doi.org/10.1016/j.apsusc.2018.06.136

Johnson, R.E., Dettre, R.H., Brandreth, D.A.: Dynamic contact angles and contact angle hysteresis. J. Coll. Interface Sci. 62(2), 205-212 (1977). https://doi.org/10.1016/0021-9797(77)90114-X

Kwok, D.Y., Leung, A., Lam, C.N.C., Li, A., Wu, R., Neumann, A.W.: Low-rate dynamic contact angles on poly(methyl methacrylate) and the determination of solid surface tensions. J. Coll. Interface Sci. 206(1), 44-51 (1998)

Lei, D., Lin, M., Li, Y., Jiang, W.: A two-angle model of dynamic wetting in microscale capillaries under low capillary numbers with experiments. J. Coll. Interface Sci. 520, 91-100 (2018). https://doi. org/10.1016/j.jcis.2018.02.074

Marmur, A., Della Volpe, C., Siboni, S., Amirfazli, A., Drelich, J.: Contact angles and wettability: towards common and accurate terminology. Surf. Innov. 5(1), 1-24 (2017). https://doi.org/10.1680/jsuin .17 .00002 
Meiron, T.S., Marmur, A., Saguy, I.S.: Contact angle measurement on rough surfaces. J. Coll. Interface Sci. 274(2), 637-644 (2004). https://doi.org/10.1016/j.jcis.2004.02.036

Moghadam, A., Jamali, M., Venkateshan, D.G., Tafreshi, H.V., Pourdeyhimi, B.: A new approach to modeling liquid intrusion in hydrophobic fibrous membranes with heterogeneous wettabilities. Coll. Surf. A 558, 154-163 (2018)

Morrow, N.R., Nguyen, M.D.: Effect of interface velocity on dynamic contact angles at rough surfaces. J. Coll. Interface Sci. 89(2), 523-531 (1982). https://doi.org/10.1016/0021-9797(82)90203-X

Morrow, N.R., Chatzis, I., Taber, J.J.: Entrapment and mobilization of residual oil in bead packs. SPE Reserv. Eng. 3(3), 927-934 (1988)

Pak, T., Butler, I.B., Geiger, S., van Dijke, M.I., Sorbie, K.S.: Droplet fragmentation: 3D imaging of a previously unidentified pore-scale process during multiphase flow in porous media. Proc. Natl. Acad. Sci. U.S.A. 112(7), 1947-1952 (2015). https://doi.org/10.1073/pnas.1420202112

Petrov, J.G., Ralston, J., Schneemilch, M., Hayes, R.A.: Dynamics of partial wetting and dewetting in welldefined systems. J. Phys. Chem. B 107(7), 1634-1645 (2003). https://doi.org/10.1021/jp026723h

Piri, M., Blunt, M.J.: Three-dimensional mixed-wet random pore-scale network modeling of two- and threephase flow in porous media. I. Model description. Phys. Rev. E: Stat. Nonlinear Soft Matter Phys. 71(2), 026301 (2005). https://doi.org/10.1103/physreve.71.026301

Quéré, D.: Wetting and roughness. Annu. Rev. Mater. Res. 38(1), 71-99 (2008). https://doi.org/10.1146/ annurev.matsci.38.060407.132434

Ramiasa, M., Ralston, J., Fetzer, R., Sedev, R.: The influence of topography on dynamic wetting. Adv. Coll. Interface. Sci. 206, 275-293 (2014). https://doi.org/10.1016/j.cis.2013.04.005

Ramos, S., Tanguy, A.: Pinning-depinning of the contact line on nanorough surfaces. Eur. Phys. J. E 19(4), 433-440 (2006). https://doi.org/10.1140/epje/i2005-10056-0

Ramos, S.M., Charlaix, E., Benyagoub, A., Toulemonde, M.: Wetting on nanorough surfaces. Phys. Rev. E: Stat. Nonlinear Soft Matter Phys. 67(3 Pt 1), 031604 (2003). https://doi.org/10.1103/PhysR evE.67.031604

Schneemilch, M., Hayes, R.A., Petrov, J.G., Ralston, J.: Dynamic wetting and dewetting of a low-energy surface by pure liquids. Langmuir 14(14), 7047-7051 (1998)

Smith, W.O., Crane, M.D.: The Jamin effect in cylindrical tubes. J. Am. Chem. Soc. 52, 1345-1349 (1930). https://doi.org/10.1021/ja01367a007

Sohrabi, M., Tehrani, D.H., Danesh, A., Henderson, G.D.: Visualization of oil recovery by water-alternating-gas injection using high-pressure micromodels. SPE J. 9(3), 290-301 (2004)

Srinivasan, V., Khandekar, S., Bouamrane, N., Lefevre, F., Bonjour, J.: Motion of an isolated liquid plug inside a capillary tube: effect of contact angle hysteresis. Exp. Fluids 56(1), 14 (2015). https://doi. org/10.1007/s00348-014-1881-7

Srinivasan, V., Sikarwar, B.S., Khandekar, S.: Experimental and simulation study on motion of an isolated liquid plug inside a dry circular capillary. In: ASME 2013 4th International Conference on Micro/ Nanoscale Heat and Mass Transfer, Hongkong, China 2013, p. 22064

Taber, J.J.: Research on enhanced oil recovery: past, present and future. Pure Appl. Chem. 52(5), 13231347 (1980). https://doi.org/10.1007/978-1-4757-0337-5_2

Uchiyama, H., Kariyasaki, A., Kagawa, M., Yamasaki, Y., Matsukuma, Y., Morooka, S.: Bubble mobilization pressure in a small-diameter tube. Chem. Eng. Technol. 38(11), 1965-1971 (2015). https://doi. org/10.1002/ceat.201500012

Publisher's Note Springer Nature remains neutral with regard to jurisdictional claims in published maps and institutional affiliations. 